\title{
Acute inferior myocardial infarction in a patient with a prosthetic aortic valve and high international normalized ratio
}

\author{
Halil Atas, Ibrahim Sari, Kenan Delil, Cigdem Ileri, Fuad Samadov \\ Marmara University Research and Training Hospital, Istanbul, Turkey
}

Postep Kardiol Inter 2014; 10, 1 (35): 63-65 DOI: 10.5114/pwki.2014.41475

\begin{abstract}
A bstract
ST elevation acute myocardial infarction in patients with a mechanical prosthetic valve is rare and usually due to inadequate anticoagulation. We present a case of acute inferior myocardial infarction in a patient with a prosthetic aortic valve and high international normalized ratio, which has not been reported previously.
\end{abstract}

Key words: myocardial infarction, prosthetic valve, international normalized ratio.

\section{Introduction}

ST elevation acute myocardial infarction (STEMI) in patients with a mechanical prosthetic valve is rare and usually due to inadequate anticoagulation [1, 2].

We present a case of acute inferior myocardial infarction in a patient with a prosthetic aortic valve and high international normalized ratio (INR), which has not been reported previously.

\section{Case report}

A 69-year-old man was admitted to emergency service with retrosternal chest pain of $2 \mathrm{~h}$ duration. His past medical history revealed aortic valve replacement and single vessel bypass surgery 4 years ago. His blood pressure was 100/60 $\mathrm{mm} \mathrm{Hg}$ and his pulse rate was 72/min. His physical examination was normal and a metallic valve sound was heard over the aortic area. His ECG showed D2, D3 aVF ST elevation and aVL, V2 reciprocal ST depression. He was on warfarin (5 mg/day), aspirin (100 mg/ day) and metoprolol (50 mg/day) therapy. His INR was unchecked for the last 3 months. Transthoracic echocardiography which was performed in the emergency department revealed segmental wall motion abnormality (inferior, inferior septum and posterior wall hypokinesia), ejection fraction of $52 \%$ and a normally functioning prosthetic aortic valve. Since the clinical scenario was com- patible with acute inferior STEMI and we were not aware about the recent INR values, he was immediately transferred to the catheterization laboratory. Coronary angiography showed a critical lesion in the proximal left anterior descending artery with a patent left internal mammary artery to left anterior descending graft flow. The circumflex artery was normal, but right coronary artery flow was impaired with a giant thrombus in the proximal segment of the vessel (Figure $1 \mathrm{~A}$ ).

We tried to aspirate the thrombus with an export catheter which resulted in suboptimal thrombus aspiration. Therefore we deployed a $3.5 \mathrm{~mm} \times 20 \mathrm{~mm}$ bare metal stent which resulted in complete flow restoration (Figure $1 \mathrm{~B}$ ). After the procedure his chest pain resolved and ECG normalized. His admission INR level was reported as 5.1. The following day his INR level was lowered to 1.8 with the help of one unit of fresh frozen plasma and the femoral sheath was removed.

On transesophageal echocardiography the prosthetic aortic valve and cardiac chambers were free of thrombus with normal function (Figure 2). The thrombosis panel revealed: factor $\mathrm{V}$ Leiden (FVL) mutation heterozygous for 1691 GA, normal methylenetetrahydrofolate reductase (MTHFR) - C677 analysis (677CC), homozygous MTHFR-A 1298 mutation (1298CC), normal FVL H1299R analysis $(1299 \mathrm{HH})$, normal Factor XIII V34L analysis (34VV), normal

\section{Corresponding author:}

Halil Atas MD, Marmara University Research and Training Hospital, Fahrettin Kerim Gökay cd, 34100 Istanbul, Turkey, phone: +905325462535, e-mail: dratashalil@gmail.com

Received: 6.11.2013, accepted: 9.01.2014. 

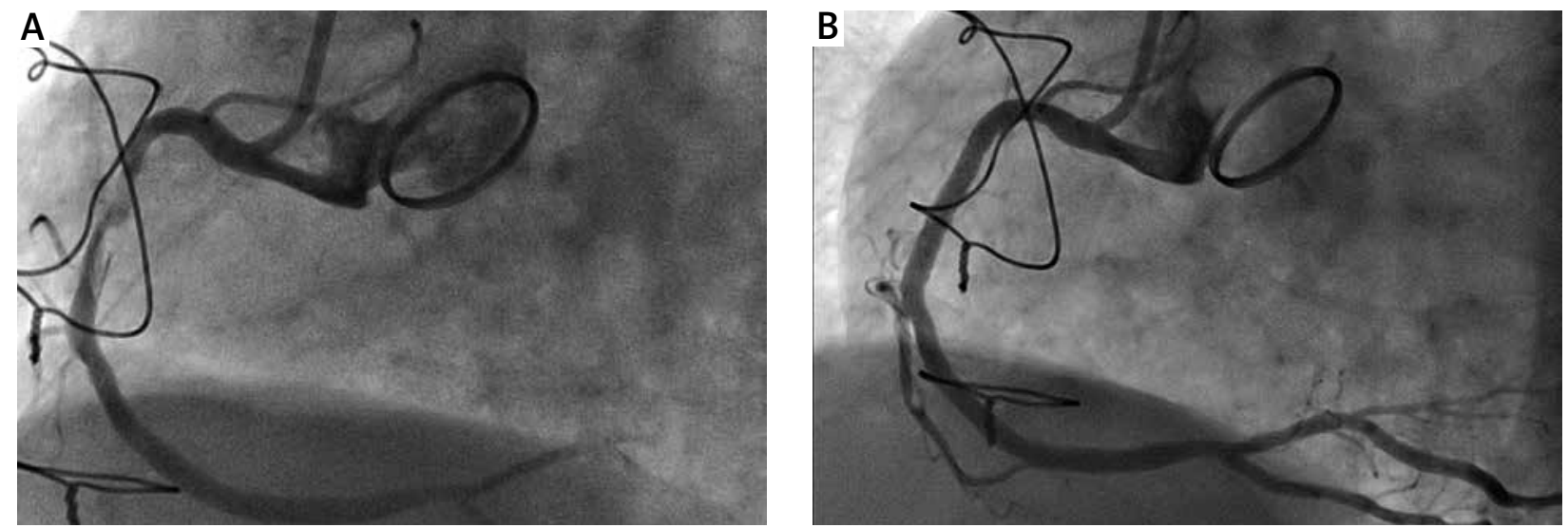

Figure 1. A - Angiographic view of the right coronary artery demonstrating impaired flow due to a giant thrombus in the proximal segment of the vessel. Note elliptic cross-sectional appearance of the prosthetic aortic valve. B - Angiographic view of the right coronary artery after deployment of $3.5 \mathrm{~mm} \times 20 \mathrm{~mm}$ BMS which resulted in complete flow restoration

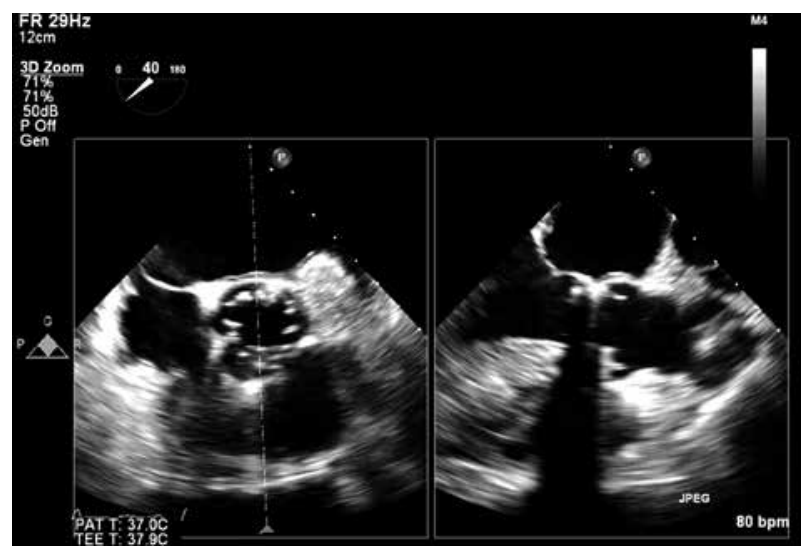

Figure 2. Transesophageal echocardiographic view of the prosthetic aortic valve demonstrating normal function without any thrombus

Beta fibrinogen-455 G>A (cardiovascular panel) analysis (455G>G) and normal Apo B R3500Q analysis (3500RR). Five days after the procedure he was discharged with clopidogrel, aspirin, warfarin, metoprolol, atorvastatin and pantoprazole.

\section{Discussion}

In the present paper, we report a case of acute inferior STEMI in a patient with a prosthetic aortic valve and an INR of 5.1, which has not been reported previously. Myocardial infarction in patients with a mechanical prosthetic valve is reported to be rare and usually due to inadequate anticoagulation. In those conditions, coronary embolism is the proposed mechanism. We have recently reported a case of myocardial infarction due to coronary embolism in a patient with prosthetic mitral valve thrombosis and an INR of 1.6 [2].

However, in the present case coronary embolism was unlikely because the INR level was high and we were not able to detect any thrombus in the cardiac chambers or around the prosthetic valve.

An interesting point of the present paper is that the patient experienced STEMI with a big thrombus burden even though he was on aspirin therapy and his INR level was already too high. We did not find such a case when we searched the literature. One possible explanation for the present case is that the patient had heterozygous factor V Leiden 1691 GA and homozygous MTHFR-A 1298 mutation (1298CC), which might have contributed to infarct formation. However, these mutations have been reported to be associated most commonly with venous thromboembolic events and the association with arterial thrombosis is less well established [3-5].

There is no consensus regarding how to treat myocardial infarction in patients with prosthetic valves. Treatment options include heparin, fibrinolysis, glycoprotein inhibitors and percutaneous coronary intervention. We performed percutaneous coronary intervention because the patient presented with typical signs and symptoms of STEMI and the subsequent high INR result precluded administration of fibrinolytic glycoprotein inhibitors.

\section{Conclusions}

Although STEMI in patients with a mechanical prosthetic valve is rare and reported to be due to inadequate anticoagulation, it may also present with high INR as in the present case.

\section{References}

1. Iakobishvili Z, Eisen A, Porter A, et al. Acute coronary syndromes in patients with prosthetic heart valves - a case series. Acute Cardiac Care 2008; 10: 148-151.

2. Yuce M, Yavuz F, Sari I, et al. A rare cause of myocardial infarction: coronary embolism in a patient with proshtetic mitral valve trombosis. East Mediterr Health J 2011; 17: 887-888. 
3. Kim RJ, Becker RC. Association between factor V Leiden, prothrombin G20210A, and methylenetetrahydrofolate reductase C677T mutations and events of the arterial circulatory system: a meta-analysis of published studies. Am Heart J 2003; 146: 948-957.

4. Juul K, Tybjaerg-Hansen A, Steffensen R, et al. Factor V Leiden: the Copenhagen City Heart Study and 2 meta-analyses. Blood 2002; 100: 3-10.

5. Doggen CJ, Cats VM, Bertina RM, et al. Interaction of coagulation defects and cardiovascular risk factors: increased risk of myocardial infarction associated with factor $\mathrm{V}$ Leiden or prothrombin 20210A. Circulation 1998; 97: 1037-1041. 